\title{
De novo transcriptome reconstruction and annotation of the Egyptian rousette bat
}

\author{
Albert K. Lee ${ }^{1,2 \dagger}$, Kirsten A. Kulcsar ${ }^{3 \dagger}$, Oliver Elliott ${ }^{2}$, Hossein Khiabanian² ${ }^{2}$ Elyse R. Nagle ${ }^{3}$, \\ Megan E.B. Jones ${ }^{4}$, Brian R. Amman ${ }^{4}$, Mariano Sanchez-Lockhart ${ }^{3}$, Jonathan S. Towner ${ }^{4}$, Gustavo Palacios ${ }^{3,5+}$ \\ and Raul Rabadan ${ }^{1,2 *+}$
}

\begin{abstract}
Background: The Egyptian Rousette bat (Rousettus aegyptiacus), a common fruit bat species found throughout Africa and the Middle East, was recently identified as a natural reservoir host of Marburg virus. With Ebola virus, Marburg virus is a member of the family Filoviridae that causes severe hemorrhagic fever disease in humans and nonhuman primates, but results in little to no pathological consequences in bats. Understanding host-pathogen interactions within reservoir host species and how it differs from hosts that experience severe disease is an important aspect of evaluating viral pathogenesis and developing novel therapeutics and methods of prevention.

Results: Progress in studying bat reservoir host responses to virus infection is hampered by the lack of host-specific reagents required for immunological studies. In order to establish a basis for the design of reagents, we sequenced, assembled, and annotated the $R$. aegyptiacus transcriptome. We performed de novo transcriptome assembly using deep RNA sequencing data from 11 distinct tissues from one male and one female bat. We observed high similarity between this transcriptome and those available from other bat species. Gene expression analysis demonstrated clustering of expression profiles by tissue, where we also identified enrichment of tissue-specific gene ontology terms. In addition, we identified and experimentally validated the expression of novel coding transcripts that may be specific to this species.
\end{abstract}

Conclusion: We comprehensively characterized the R. aegyptiacus transcriptome de novo. This transcriptome will be an important resource for understanding bat immunology, physiology, disease pathogenesis, and virus transmission.

Keywords: RNA-seq, Transcriptome, Genomics, Annotation, Database

\section{Background}

Bats (order: Chiroptera) constitute an abundant and diverse mammalian lineage comprising approximately $20 \%$ of all known mammalian diversity [1]. Bats have evolved apart from other mammals for more than 50 million years [2] and are divided into two major suborders; the Yinpterochiroptera (megachiroptera) and the Yangochiroptera (microchiroptera). Yinpterochiroptera includes the family Pteropodidae and genera Rousettes

\footnotetext{
*Correspondence: rr2579@cumc.columbia.edu

${ }^{\dagger}$ Equal contributors

${ }^{1}$ Department of Biomedical Informatics, Columbia University College of Physicians and Surgeons, 1130 St. Nicholas Ave, New York, USA

2 Department of Systems Biology, Columbia University College of Physicians and Surgeons, 1130 St. Nicholas Ave, New York, USA

Full list of author information is available at the end of the article
}

and Pteropus whereas Yangochiroptera includes the family Myotidae and genus Myotis [3]. Unlike most mammals, bats can fly and this ability enabled their wide geographical range and increased metabolism [2]. Interestingly, bats have recently come to the forefront of zoonotic disease research with vast number of pathogens identified in a wide variety of bat species [2].

Upwards of 85 different viruses, primarily RNA viruses, have been detected and/or isolated from bats [2, 4]. Amongst these are emerging viruses that cause lethal disease in humans and nonhuman primates including Nipah virus [5, 6], Hendra virus [7], severe acute respiratory syndrome (SARS)-like coronavirus [8], Middle East respiratory syndrome coronavirus (MERS-CoV) [9], Marburg virus (MARV) [10-13], and Ebola virus (EBOV) [14-16]. Despite the severe virulence of these viruses in humans, 
infected bats are often asymptomatic [13, 17-22]. Nipah virus and Hendra virus interactions with their natural reservoir hosts, Pteropus vampyrus and Pteropus alecto, respectively, are well characterized. Experimental infections of bats with high doses of henipaviruses have shown virus replication and shedding with little to no disease [20-22]. Remarkably, the only viruses known to have induced any observable pathology in bats are rabies virus and Australian bat lyssavirus [2, 23]. Understanding mechanisms of disease and differential responses to infection in asymptomatic reservoir host species compared to species that exhibit severe pathology will help inform the development of novel therapeutics and disease prevention approaches.

Rousettus aegyptiacus, commonly known as the Egyptian rousette bat, has been identified as a natural reservoir host for MARV through ecological, epidemiological, and experimental studies [10, 12, 13, 18, 19, 24]. Furthermore, it has been speculated this bat could host Ebola virus [12, 25-27], although recent experimental infection studies have shown Ebola virus does not replicate well in $R$. aegeyptiacus [28]. The majority of human outbreaks due to MARV have been associated with caves inhabited by $R$. aegyptiacus. Furthermore, epidemiological surveillance of the $R$. aegyptiacus colony located in the Python cave in Uganda revealed a biannual spike in Marburg virus prevalence. This pattern correlated strongly with spillover transmission events in humans [24]. Initial studies in captive bats evaluated clinical signs, virus dissemination, and virus shedding patterns during experimental infection with a MARV isolate derived from wild bats [13]. Consistent with a natural reservoir host, the bats showed little to no evidence of disease even though the virus disseminated throughout their body and was actively shed [13]. These results were confirmed when bats were infected with MARV Angola, a strain isolated from a lethal human case [18]. In the absence of genetic and transcriptomic information for $R$. aegyptiacus and with limited available reagents, studying this reservoir host animal model has been challenging.

The rapid expansion in genomic knowledge for different bat species has facilitated comparative studies that rely on the identification of genes and gene families, and has established a framework for developing necessary reagents. Full genome annotations for Pteropus vampyrus (2.63X, [29]), Myotis lucifugus (6.6X, [29]) Pteropus alecto (110x, [30]), Myotis davidii (110x, [30]), and Myotis brandtii (77.8X, [31]) are now available. Additionally, transcriptomic annotations for Pteropus alecto [32] and Artibeus jamaicensis [33] have been published. In particular, the complementary genome and transcriptome annotations for $P$. alecto has aided studies on henipavirus infections in its reservoir host [30, 32]. The host transcriptional response to different viruses was also recently assessed in a kidney cell line derived from $P$. vampyrus utilizing the previously annotated genome [34].

In this manuscript, we report the transcriptomic annotation of $R$. aegyptiacus from a de novo assembly of RNA sequencing data from 11 tissues isolated from a male and a female bat. We identified 24,118 canonical coding transcripts whose expression profiles were consistent with the corresponding tissues of origin. In addition, we identified and validated novel coding transcripts that do not have any homology with the known sequences. Furthermore, we evaluated the annotation for immune-related genes and assessed the presence and expression of genes associated with a variety of immune functions.

\section{Results and discussion}

\section{De novo transcriptome assembly of $R$. aegyptiacus}

We employed a de novo assembly approach to generate a comprehensive transcriptome without relying on a genome reference. First, we generated 20 RNA-seq libraries consisting of 11 tissue types (Table 1, Fig. 1a) each collected from one male and one female $R$. aegyptiacus bat, which yielded approximately 2.1 billion reads. We then assembled the high quality reads using Trinity [35] (Fig. 1b). This process generated 14,796,219 contigs. The assembly had high continuity and coverage with a median number of 718,807 contigs and median N50 of 1,540

Table 1 Library Information and Assembly Statistics

\begin{tabular}{lllllll}
\hline Bat & Gender & Tissue & Read count & Library & N50 & Number of contigs \\
\hline BAT01 & F & BM & 67896687 & single & 1736 & 609943 \\
BAT02 & F & BR & 55004118 & single & 884 & 896445 \\
BAT03 & F & HT & 77315750 & single & 1263 & 717588 \\
BAT04 & F & KY & 59782352 & single & 1174 & 720026 \\
BAT05 & F & LG & 77510852 & paired & 1822 & 903831 \\
BAT06 & F & LN & 63170354 & single & 1566 & 638083 \\
BAT07 & F & LV & 89970603 & paired & 1566 & 697125 \\
BAT08 & F & OV & 75051316 & single & 1401 & 875888 \\
BAT09 & F & PB & 56553369 & single & 1890 & 404332 \\
BAT10 & F & SP & 56141808 & single & 1340 & 716771 \\
BAT11 & M & BM & 47988156 & paired & 1808 & 744115 \\
BAT12 & M & BR & 75378417 & paired & 1490 & 1088331 \\
BAT13 & M & HT & 20042200 & paired & 748 & 497729 \\
BAT14 & M & KY & 71478010 & paired & 1514 & 872829 \\
BAT15 & M & LG & 15525010 & paired & 668 & 575991 \\
BAT16 & M & LN & 88471565 & paired & 2186 & 797125 \\
BAT17 & M & LV & 27358079 & paired & 925 & 431513 \\
BAT18 & M & PB & 92707184 & paired & 1745 & 556053 \\
BAT19 & M & SP & 98465277 & paired & 2141 & 873259 \\
BAT20 & M & TT & 96476242 & paired & 1866 & 1179242 \\
\hline & & & & & &
\end{tabular}




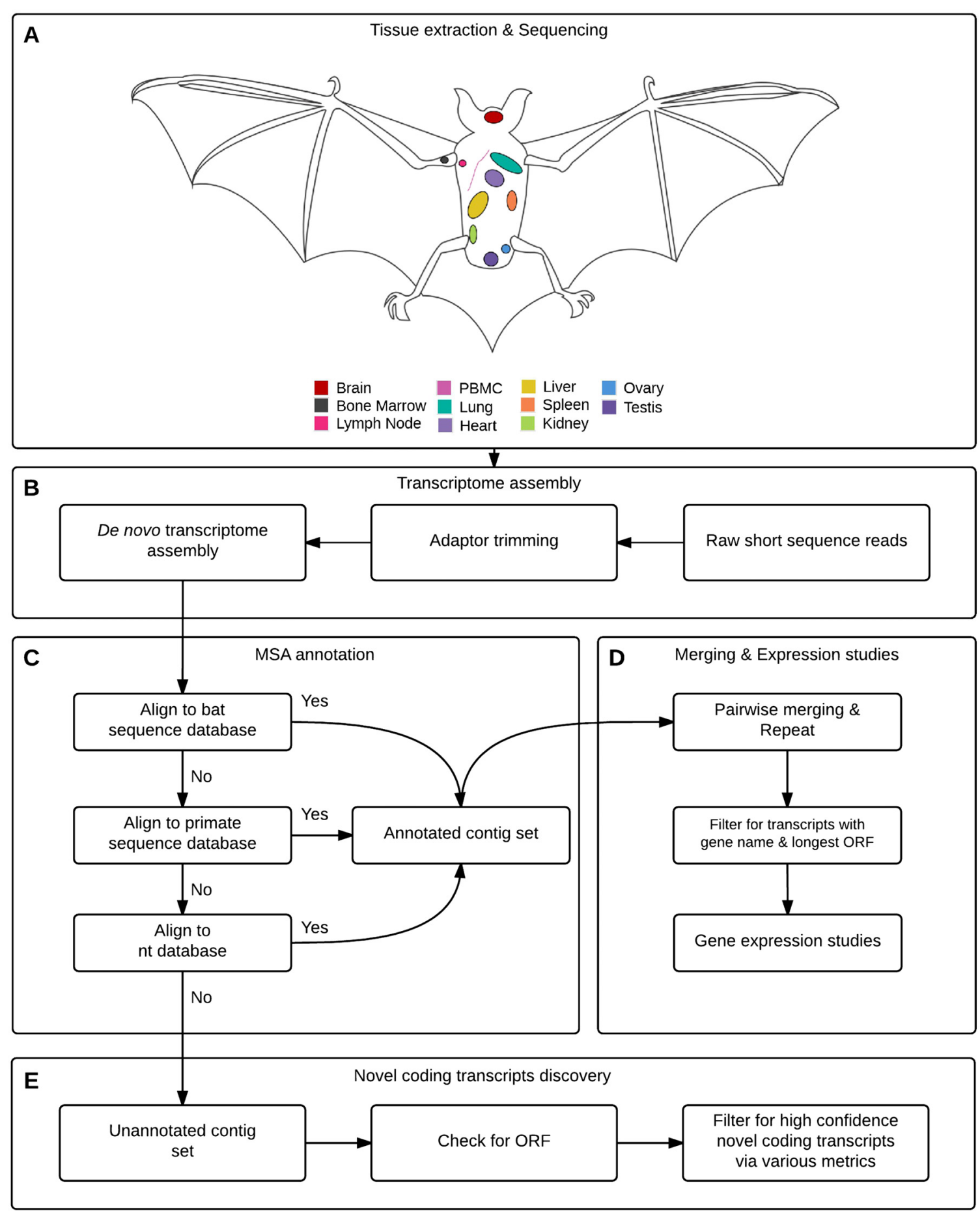

Fig. 1 Schematic of the de novo transcriptome reconstruction and analysis pipeline. The pipeline consists of 5 steps. a Data generation: Multiple tissues are extracted from R. aegyptiacus and sequenced. b De novo Transcriptome assembly: Individual samples are first preprocessed to remove adapter sequences and assembled into contigs de novo. c MSA annotation: Once the set of contigs is generated, they are annotated using BLAST against three databases. In each step, unannotated contigs are iteratively annotated using the downstream databases. $\mathbf{d}$ Mering and Expression studies: A nonredundant contig set is obtained by merging the contig set of individual tissues two at a time. This pairwise merging is repeated until only one contig set is left. The subset of this contig can be obtained for the downstream analysis such as gene expression analysis by taking the transcripts with gene symbol and ORF sequence. See Fig. 2 for details. e Discovery of Novel Coding Transcripts: Novel coding transcripts can be identified by searching for contigs that failed annotation in the previous steps. Various metrics can be applied to generate high confidence novel coding transcript candidates 
across all tissues (Table 1). To comprehensively annotate the contigs, we used the Multiple Species Annotation (MSA) pipeline [36], which leverages the homology of known sequences of related species. We assigned gene symbols to contigs when this information was available. This process clustered the contigs into isoform groups (Fig. 1c).

\section{R.aegyptiacus transcriptome captures a majority of bat transcripts}

We compared our assembly to the transcriptomes of three related bat species -- M. davidii, P. alecto, and $M$. brandtii. Using BLAST, we recovered $90.1 \%$ of $M$. davidii transcripts, $89.54 \%$ of $M$. brandtii transcripts, and $97.38 \%$ of $P$. alecto transcripts. This result is consistent with the evolutionary history of these bats considering that $P$. alecto and $R$. aegyptiacus belong to the same family of Pteropodidae.

\section{Combining the transcriptome to generate nonredundant contigs}

Tissue-specific transcriptome assemblies contained different numbers of contigs, due to their different levels of expression and sequencing depth. Without a common ground for comparison, it was difficult to perform downstream comparative analyses such as differential gene expression analysis; therefore, we combined contigs from all tissues into one unified, nonredundant reference transcriptome (Fig. 1d). To this end, we iteratively merged the assemblies two at a time, similar to the approach employed in [37] (Fig. 1d). We obtained 4,746,293 nonredundant contigs. Among the nonredundant contigs, $974,765(20.54 \%)$ of the sequences were annotated by bat sequences, $860,578(18.13 \%)$ by primate sequences, and 104,796 (2.2\%) by sequences in nt database (Fig. 2a). The nonredundant contig set had slightly lower sensitivity, though it still remained high; $86.60 \%$ of $M$. davidii, $85.95 \%$ of $M$. brandtii, and $95.30 \%$ of P. alecto transcripts were recovered. The resulting annotated contigs were assigned gene names and combined using the longest annotated ORF as the transcript. This resulted in an annotation for $R$. aegyptiacus that contained a total of 24,118 genes. To determine the efficiency of using the MSA pipeline, we determined that $84 \%$ (20,207 genes) of the contigs were annotated using the bat database and $16 \%(3,911$ genes) were subsequently annotated using the primate database. These data show that the MSA pipeline, which utilizes known transcripts from related species only, is a sensitive and efficient method for de novo transcriptome annotation.

\section{Biological validity via expression analysis}

We evaluated biological validity of the reconstructed transcriptome by analyzing global expression patterns across

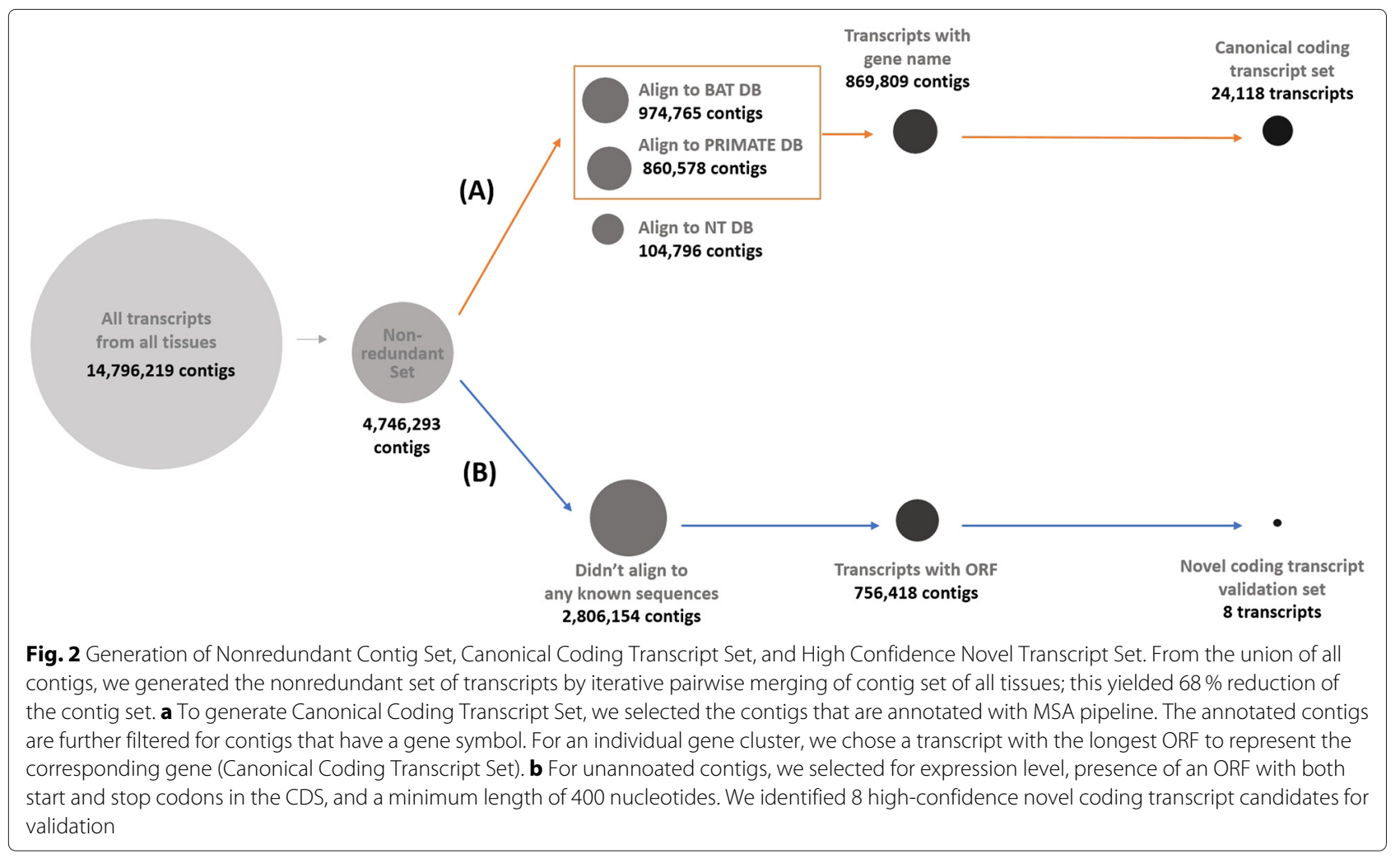


the different tissues. If the transcriptome assembly and annotations were accurate, the expression profiles of a given tissue should cluster with those of the same tissue origin and segregate from those of different origins $[36,38]$. A gene can result in more than one transcript isoform; therefore, to capture the highest amount of information, for each gene, we focused on the transcript with the longest open reading frame (ORF) (Fig. 2a). After normalizing the expression values, we performed Multidimensional Scaling (MDS) to determine the relationships between the gene expression patterns in different tissues. As expected, MDS showed a clear separation of the samples according to the tissue of origin (Fig. 3a) and explains $74 \%$ of the variance in the data. To examine the evolutionary relationship among tissues, we performed hierarchical clustering of the gene expression profiles (Fig. 3b). The brain, which has a different developmental pathway compared to the other organs, was classified as an outgroup. The spleen, lymph node, and bone marrow are all organs of the immune system and, as expected, clustered near each other. The peripheral blood contains some of the same cell types as the immune organs, thus, clustered near these tissues. Lastly, the gonads and kidney, which develop from the intermediate mesoderm, were grouped as neighbors in the tree. These results suggest that our transcriptome captured sufficient heterogeneity of gene expression to distinguish individual tissues while preserving their developmental relationships.

\section{Gene Ontology analysis}

We further assessed biological validity of our transcriptome assembly through gene Ontology (GO) analysis of tissue-specific expression profiles. We compared expression profile of each tissue with the average expression in the whole dataset, and identified the top 200 most differentially expressed genes based on a generalized linear modeling framework. Using this list, we examined the enriched GO biological process (BP) terms. Figure 4 shows the top $10 \mathrm{GO}$ BP terms from the bone marrow, spleen, lymph nodes, and peripheral blood mononuclear cells (PBMCs). (For other tissues, see Additional file 1). Terms enriched for each tissue are consistent with their expected physiological functions.

\section{Identification of immune-related transcripts}

$R$. aegyptiacus is a natural reservoir host for MARV, allowing for virus replication and dissemination with little to no pathological consequences [13, 17-22]. One important aspect of reservoir host biology is how their immune response compares to that of animal species that experiences severe disease, such as humans. Therefore, we examined the transcriptome for the presence of immune-related genes. We associated the $\mathrm{R}$. aegyptiacus gene set with GO terms based on the human-specific gene

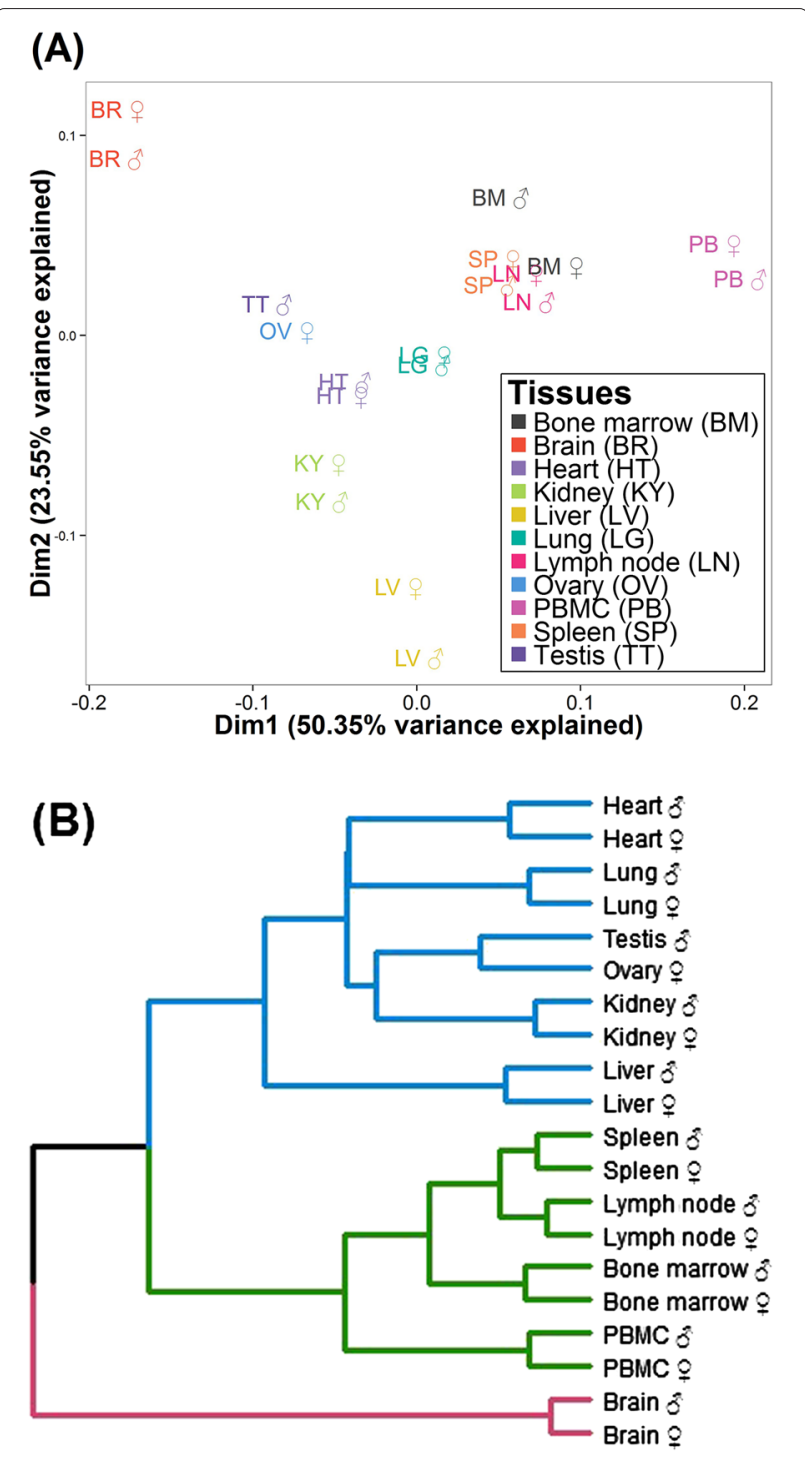

Fig. 3 MDS of Gene Expression Profiles of Bat Tissues. a We assessed the biological validity and quality of our transcriptome annotations by performing Multidimensional Scaling (using 1-spearman correlation as distance) on gene expression profiles of all tissues using the 22,398 genes as feature vector. The first two coordinates explained $73.9 \%$ of the variance in the data. $\mathbf{b}$ We performed hierarchical clustering of expression profiles using 1-spearman correlation as distance. The clustering suggested presence of three groups that correspond to separate developmental origins. Tissues used are Bone (BM), Brain (BR), Heart (HT), Kidney (KY), Liver (LV), Lung (LG), Lymph (LN), Ovary (OV), PBMC (PB), Spleen (SP), Testes (TT) of the male (M) and female (F) bat

ontology annotation. This resulted in 14,781 genes that mapped to 14,817 GO terms. We used CateGOrizer [39] and applied the immune class GOSlim terms to identify immune-related genes from this set. Similar to previous studies in P. alecto and A. jamaicensis, we found that out of 14,817 GO terms, approximately $2.75 \%$ were associated with immune response [32, 33]. Amongst the most represented GO terms were cytokine production, lymphocyte 


\begin{tabular}{|c|c|}
\hline Bone Marrow & Spleen \\
\hline 1. (GO:0002376) immune system process & 1. (GO:0019752) carboxylic acid metabolic process \\
\hline 2. (GO:0009611) response to wounding & 2. (GO:0043436) oxoacid metabolic process \\
\hline 3. (GO:0050832) defense response to fungus & 3. (GO:0006082) organic acid metabolic process \\
\hline 4. (GO:0007596) blood coagulation & 4. (GO:0055114) oxidation-reduction process \\
\hline 5. (GO:0050817) coagulation & 5. (GO:0009063) cellular amino acid catabolic process \\
\hline 6. (GO:0007599) hemostasis & 6. (GO:0044282) small molecule catabolic process \\
\hline 7. (GO:0050878) regulation of body fluid levels & 7. (GO:0016054) organic acid catabolic process \\
\hline 8. (GO:0042060) wound healing & 8. (GO:0046395) carboxylic acid catabolic process \\
\hline 9. (GO:0007155) cell adhesion & 9. (GO:0044281) small molecule metabolic process \\
\hline 10. (GO:0022610) biological adhesion & 10. (GO:0006520) cellular amino acid metabolic process \\
\hline Lymph node & PBMC \\
\hline 1. (GO:0046649) lymphocyte activation & 1. (GO:0007155) cell adhesion \\
\hline 2. (GO:0045321) leukocyte activation & 2. (GO:0022610) biological adhesion \\
\hline 3. (GO:0001775) cell activation & 3. (GO:0006935) chemotaxis \\
\hline 4. (GO:0031295) T cell costimulation & 4. (GO:0042330) taxis \\
\hline 5. (GO:0031294) lymphocyte costimulation & 5. (GO:0030198) extracellular matrix organization \\
\hline 6. (GO:0002376) immune system process & 6. (GO:0043062) extracellular structure organization \\
\hline 7. (GO:0002682) regulation of immune system process & 7. (GO:0006928) cellular component movement \\
\hline 8. (GO:0002694) regulation of leukocyte activation & 8. (GO:0009605) response to external stimulus \\
\hline 9. (GO:0042110) T cell activation & 9. (GO:0040011) locomotion \\
\hline 10. (GO:0050865) regulation of cell activation & 10. (GO:0050896) response to stimulus \\
\hline
\end{tabular}

Fig. 4 Top Ten Enriched Gene Ontology Biological Process Terms for bone marrow, spleen, lymph node, and PBMC. In each panel, the terms are listed in descending order of significance of enrichment. These tissues, in particular are associated with different aspects of the immune system and these associations are observed within the GO BP terms identified

activation, $\mathrm{T}$ cell activation, regulation of apoptosis, and regulation of lymphocyte activation (Fig. 5).

We next searched for specific genes related to various aspects of the immune response in other mammals, primarily mice and humans. We first evaluated the annotation of the transcriptome for the presence of anti-viral genes. A multitude of pattern recognition receptors were identified including toll-like receptors (TLRs) 1-9, RIGI, MDA5, and LGP2 along with the important scaffold and signaling molecules Myd88 and MAVS. A variety of antiviral molecules were also found, including Mx1 and Mx2, PKR, STING, IRF3, IRF5, IRF7, members of the IFIT and IFITM families, and ISG15. We also looked for the presence of type I, II, and III interferons (IFN). We were able to identify IFNgamma, IFNgamma2, and IFNalpha. Transcripts corresponding to the IFN receptor subunits IFNAR1 and IFNAR2 were also identified. IFNalpha and IFNbeta have been previously characterized by cloning from stimulated cells [40]. We, however, did not find any contigs corresponding to IFNB. To eliminate the possibility of an impaired assembly, we aligned the processed RNA-seq reads to the IFNB sequence from P. alecto [41] (Additional file 2 and Additional file 3). We detected only 2 reads from $R$. aegyptiacus, which did not provide sufficient coverage to construct the transcript. These data suggest that IFNB expression in healthy tissues of $R$. aegyptiacus is low, consistent with other mammals in which IFNB is primarily expressed after exposure to a stimulus.

We also searched the transcriptome for genes associated with innate immune cells. We found the transcripts for the CD14 and CD11c genes, which are commonly used for phenotyping macrophages and dendritic cells, as well as transcripts for the CD80 and CD86 genes, which are useful for evaluating the activation status of these cells. Genes associated with natural killer (NK) cells, however, were less evident. We were able to identify transcripts of co-receptor gene CD56, but not CD16. Transcripts of genes encoding for molecules in the killer cell lectin-like receptor (KLR) family, including NKG2A and NKG2D, were also not found. In other bat transcriptomes, such as $P$. alecto and A. jamaicensis, coverage of NK cellrelated genes was more sparse than that of other mammals $[32,33]$. A similar observation was made in the genome of $M$. davidii [30]. The absence of NK cell-related genes in the $R$. aegyptiacus transcriptome further strengthens the theory that bats might contain a different NK cell receptor repertoire than other species.

Next, we examined the repertoire of genes associated with adaptive the immune response. We identified a variety of transcripts associated with $\mathrm{T}$ cell identification, activation, inhibition, and differentiation including $\mathrm{CD} 3 \epsilon$, CD4, CD8a, CD25, CD69, CCR7, PD-1, CTLA4, GATA3, foxp3, and Tbet. Interestingly, we were able to identify transcripts for the TCR $\alpha$ and $\operatorname{TCR} \beta$ chains, but were unable to find transcripts for the TCR $\delta$ and TCR $\gamma$ chains. The transcriptome annotation for $P$. alecto included these genes, but they were present at low levels [32]. This supports the notion that $\alpha \beta \mathrm{T}$ cells are the predominant $\mathrm{T}$ cell subset in bats. We also looked at genes associated with B cells and were able to find transcripts for CD19, CD20, CD27, as well as transcripts that were similar to 


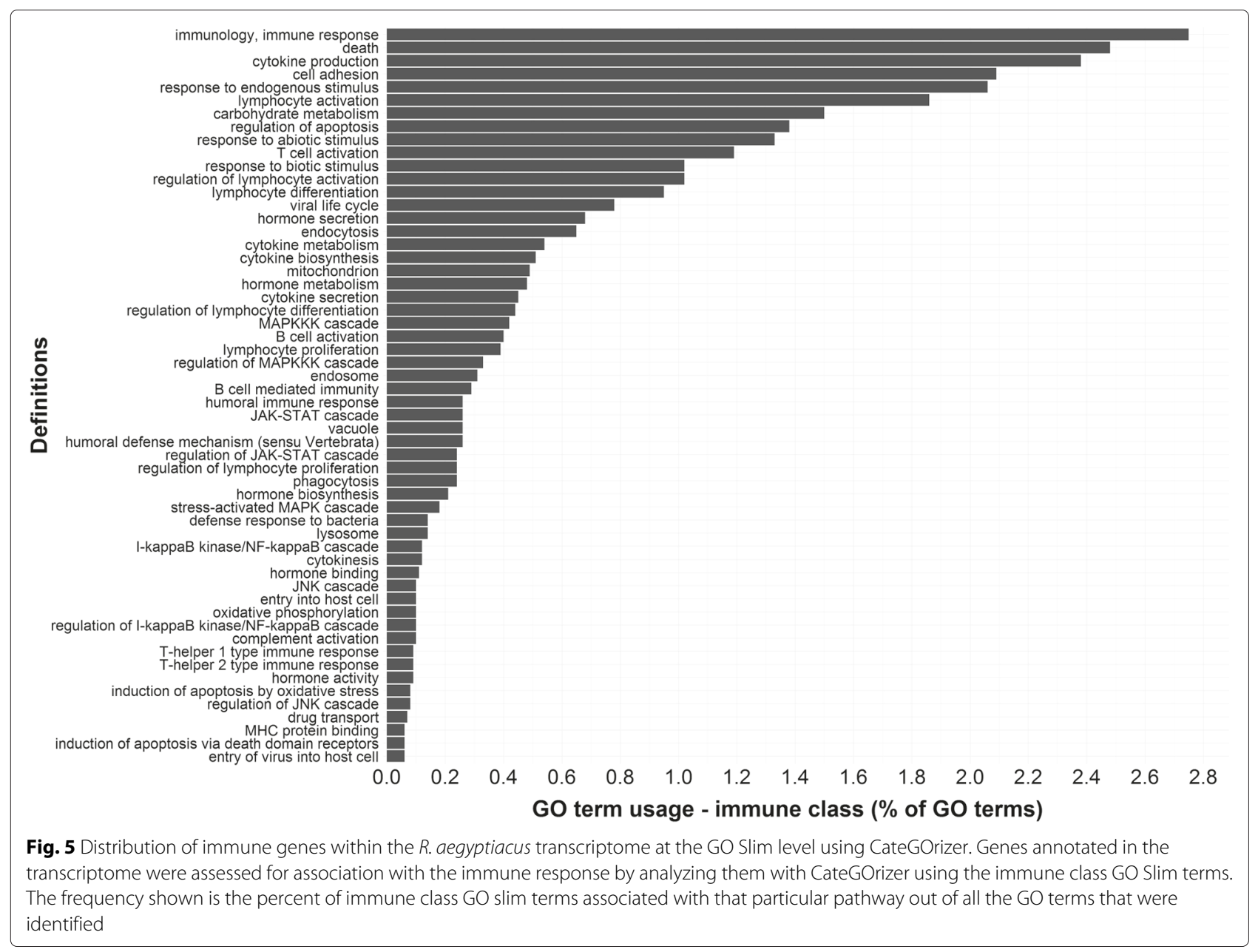

the immunoglobulin heavy chains A, E, G, and M and the immunoglobulin light chains $\kappa$ and $\lambda$. Future analysis of the $R$. aegyptiacus genome is required to fully evaluate the immunoglobulin gene repertoire.

Finally, we studied the cytokine and chemokine repertoire, important for shaping both innate and adaptive immune responses. We found a variety of transcripts corresponding to a wide array of both pro-inflammatory and anti-inflammatory cytokines. These included IL-2, IL-4, IL-5, IL-6, IL-12a, IL-12b, IL-17a, IL-23, IL-10, TGF $\beta$, TNF, IFN $\gamma$, IL- $1 \beta$, CCL2, CCL5, and CXCL10. Altogether, the reference transcriptome generated for $R$. aegyptiacus provides an excellent foundation for investigating reservoir host immunology in bats.

\section{Novel transcripts}

There were 2,806,154 unannotated contigs from the nonredundant contig set (Fig. 2b). Of those, $71.6 \%$ $(2,008,503$ contigs) did not have an ORF suggesting the majority of these contigs may be noncoding transcripts. To determine if the unannotated contigs were real or artifacts from the assembly, we used BLAST to align this set of contigs to the P. alecto genome and found that $96 \%$ $(2,706,432$ contigs) were aligned. To evaluate the possibility of an incomplete or impaired assembly, we grouped the aligned contigs into a total of 1,012,664 clusters based on the presence of overlapping sequences. This reduction suggests that multiple isoform expression patterns between different tissues may have affected our assembly or that our short read assembly may have been incomplete. Nonetheless, the number of unannotated contigs that aligned to the $P$. alecto genome suggests that these contigs, either coding or noncoding, may be novel transcripts shared within the order Pteropodinae. Future studies evaluating the conservation and possible functions of these sequences are essential to determine the importance of these genetic elements. To validate novel contigs in $R$. aegyptiacus that appeared to be coding we utilized PCR. Primers were designed to produce amplicons for eight highly expressed, unannotated contigs that contained ORFs longer than $400 \mathrm{bp}$. Using RNA isolated from the spleen, we were able to produce amplicons of the expected size from at least one bat (Fig. 6 and Additional file 4). The sequences of these amplicons were found to 


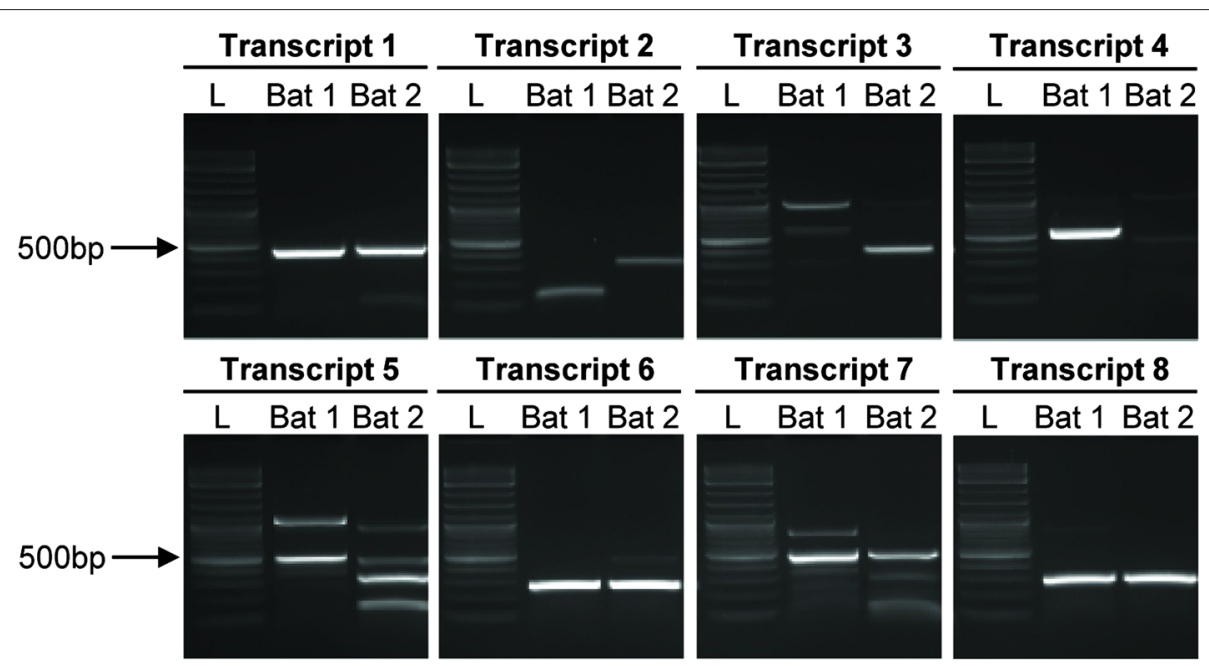

Fig. 6 Unannotated, novel transcripts from R. aegyptiacus were validated of by RT-PCR. RNA from the spleen of both bats was reverse transcribed to make cDNA. The cDNA was amplified using primers specific for one of 8 novel transcripts that were unannotated in the assembly, but contained a complete ORF larger than 400 nucleotides. The expected product sizes were: transcript 1, 457 bp; transcript 2, 450 bp; transcript 3, 419 bp; transcript 4, 548 bp; transcript 5, 469 bp, transcript 6, 277 bp; transcript 7, 507 bp; and transcript 8, 301 bp

match the expected sequence from the assembled ORF of the unannotated contig. These contigs also showed high sequence similarity to the $P$. alecto genome. In particular, six of the 8 validated transcripts showed sequence similarity higher than $75 \%$ at a query coverage greater than $64 \%$. The other two validated transcripts had a query coverage of 23 with $78.36 \%$ identity (transcript 1 in Fig. 6) and a query coverage of 7 with $91.27 \%$ identity (transcript 2 in Fig. 6) (Additional file 5); therefore, we hypothesize that these transcripts might be specific to $R$. aegyptiacus. Further investigation is needed to fully understand the characteristics and biological functions associated with the proteins these contigs encode.

\section{Conclusion}

In this paper, we presented the comprehensively annotated of transcriptome of $R$. aegyptiacus and assessed its quality and biological validity. This transcriptome will be an important resource to study bat immunology. In particular, it will facilitate the process of investigating differences in host responses between asymptomatic reservoir host species and species that exhibit severe pathology. It will also pave the way for the development of novel therapeutics and prevention approaches against emerging zoonotic virus outbreaks.

\section{Methods}

\section{Sample preparation}

Tissues and blood were collected from one male and one female adult $R$. aegyptiacus bats that were bred and housed at the colony established at the Center for Disease Control and Prevention, Atlanta, GA, USA
(Amman et al. 2015 [13]). Approximately $100 \mathrm{mg}$ of the following tissues were collected and homogenized in $1 \mathrm{~mL}$ of Trizol LS (Invitrogen, Carlsbad, CA): liver (bat id:BAT7, BAT17), lung (BAT05, BAT15), heart (BAT03, BAT13), kidney (BAT04, BAT14), brain (BAT02, BAT12), axillary lymph nodes (bilateral, pooled) (BAT06, BAT16), spleen (BAT10, BAT19), bone marrow (BAT01, BAT11), and gonad (BAT08, BAT20). PBMCs (BAT08, BAT18) were isolated from the blood and stored in Trizol LS as well.

RNA was extracted using the PureLink RNA Mini kit (Invitrogen, Carlsbad, CA). cDNA was synthesized using the TruSeq Stranded Total RNA Sample Prep Kit (Illumina, San Deigo, CA) according to the manufacturer's protocol. The libraries were evaluated for quality using the Agilent 2100 Bioanalyzer (Agilent, Santa Clara, CA). After quantification by real-time PCR with the KAPA qPCR Kit (Kapa Biosystems, Woburn, MA), libraries were diluted to $10 \mathrm{nM}$. Cluster amplification was performed on the Illumina cBot and libraries were sequenced on the Illumina HiSeq 2500. Eight of the female bat libraries were single-end, while the remaining tissues from the female bat and all tissues from the male bat were paired-end. All of the libraries sequenced were $125 \mathrm{bp}$ in length. The average library depth was $66 \mathrm{M}$ reads (minimum $16 \mathrm{M}$ and maximum $98 \mathrm{M}$ ).

\section{Ethics statement}

All experimental procedures were conducted with approval from the Centers for Disease Control and Prevention (CDC, Atlanta, GA, USA) Institutional Animal Care and Use Committee, and in strict accordance with the Guide for the Care and Use of Laboratory Animals 
(Committee for the Update of the Guide for the Care and Use of Laboratory Animals 2011). The CDC is an Association for Assessment and Accreditation of Laboratory Animal Care International fully accredited research facility. No human patient-derived clinical materials were used in these studies.

\section{De novo transcriptome assembly}

We first examined the quality of the reads using FastQC v0.11.3 [42]. We also preprocessed the reads to remove the adapter sequence using cutadapt v1.5 [43]. We removed "AGATCGGAAGAGCACACGTCTGAACTCC AGTCAC" from the forward strand and "AGATCGGAAGAGCGTCGTGTAGGGAAAGAGTGT-AGATCTCGGTGGTCGCCGTATCATT" from the reverse strand. We performed strand-specific de novo transcriptome assembly using Trinity r20140413p1 [35] with the parameters: “-normalize_reads" and "-SS_lib_type FR", along with its default parameters for all of our samples.

\section{Homology based annotation of the transcriptome}

For annotation of contigs and clustering them into a gene model, we used Multiple Species Annotation pipeline, an nucleotide-based annotation approach that is more efficient and faster than BLASTX [36]. To make a BLAST [44] database for bats, we started with the complete "Nucleotide collection" (nt) database. We exported all accession numbers of the bat sequences at NCBI and made a subset database from nt using "blastdb_aliastool -db nt -dbtype nucl -gilistbats.sequence.gi.txt -title Bats -out Bats". Using the same type of query, we also created a database for primates including humans due to their extraordinarily well-annotated transcriptomes, which will maximize the power of our annotation pipeline. We then used BLAST to iteratively align the contigs to the bat $\mathrm{db}$, the primate $\mathrm{db}$, and finally nt using a subtractive approach: what did not align to the bat db was aligned to the primate $\mathrm{db}$, and what did not align to the primate $\mathrm{db}$ was aligned to nt.

\section{Sensitivity of R.aegyptiacus transcriptome}

To assess the coverage of our transcriptome, we downloaded the $M$. davidii, $P$. alecto, and $M$. brandtii transcriptomes from NCBI Eukaryotic genomes annotations [41]. We generated a BLAST index out of union of all contigs from our samples, and aligned the three bat contigs to our BLAST databases. We chose the alignment with $70 \%$ of sequence identity with maximum evalue of $1 \mathrm{e}-4$.

\section{Nonredundant transcriptome assembly}

To generate a nonredundant set of contigs, we iteratively merged individual assemblies using the the methods similar to the [37] employed to merge the kmers. Using CD-HIT-EST v4.6 [45] with sequence identity threshold of 0.99 , we merged the first two pairs of contig sets (of sample $i$ and sample $i+1$ ) upto the final sample $n$. After each iteration, we merged the resulting merged contig sets using a similar approach until only one contig set remained.

\section{Canonical coding transcript set}

For the expression profiling, we generated a reference transcriptome consisting of transcripts each representing a gene model according to the following method: We first used TransDecoder (r20140413p1) [46] to find the ORF of all transcripts. Then, based on the MSA pipeline, we chose a transcript with gene symbols and the longest ORF in each gene cluster to capture the most information for downstream expression analysis. We did not consider the contigs mapped to nt database in this manuscript because obtaining feature files for all sequences as required by the MSA pipeline was computationally impractical, and a majority of the gene symbols $(24,118)$ are captured in the bat and primate databases.

\section{Gene expression and gene ontology analysis}

After a canonical transcript set was obtained, we used this as a transcriptome reference for expression analysis. We mapped the preprocessed reads to this reference using RSEM v1.2.19 [47] and obtained a gene-to-count matrix. We removed the transcripts with expression variance equal to zero or with low expression (count $<=$ 10). For MDS plot, we used the spearman correlaton as a distance measure and "cmdscale" from the "stats" package in $\mathrm{R}$ [48]. To explore the biological processes in each gene expression profile, we employed a oneto-all sample comparison using the EdgeR generalized linear model framework $[49,50]$. For each tissue, we compared individual gene expression within the tissue versus the average expression of all other tissues. With each tissue having differently ranked gene lists, we then selected top 200 genes and ran gene ontology analysis using topGO [51] with human-specific gene ontology annotation [52].

\section{Analysis of unannotated transcripts and identification of novel transcripts and validation}

We used BLAST [44] to align unannotated contigs to the genome of $P$. alecto with the evalue of $1 \mathrm{e}-4$ and query coverage of $40 \%$. To cluster the aligned contigs into groups, we used bedtools [53] setting the distance threshold parameter at 0 . For transcripts that did not align with any similarity to bat, primate, or nt BLAST databases, we applied a series of filters to select for the coding transcripts to be validated. We used the following criteria: an ORF that was complete with both a start and stop codon, an ORF that was at least $400 \mathrm{bp}$ in size, and a transcript that was expressed (a read count $>0$ ). We further 
selected for the novel transcripts with usuable primers using primer-BLAST [54]. Using these criteria, the number of novel transcripts was narrowed down to a total of 8. The primers and expected amplicon size are listed in Additional file 4.

For validation, RNA was extracted from the spleen tissue of both the male and female bats using Trizol LS (Invitrogen, Carlsbad, CA). cDNA was synthesized from $2.5 \mu \mathrm{g}$ of RNA using the Superscript III First-strand Synthesis SuperMix (Invitrogen, Carlsbad, CA). Amplicons for each of the primer sets were generated using Phusion HotStart Flex DNA polymerase (New England BioLabs, Ipswitch, MA) and run on a $1.5 \%$ agarose gel for visualization. The correct size amplicon was gel extracted, quantified, and Sanger sequenced on the Applied Biosystems $3730 \times 1$ DNA Analyzer.

\section{Additional files}

Additional file 1: Gene Ontology analysis of all tissues. Three sheets contain enriched GO terms of Biological Process, Molecular Function, and Cellular Compartment in individual tissues. (XLS $31 \mathrm{~kb}$ )

Additional file 2: Alignment of $R$. aegyptiacus reads to $P$. alecto transcripts. The preprocessed reads are aligned to the interferon and immunoglobulin transcripts of P. alecto obtained from [41] and [32]. The sequences used are described in Annotation file 2. (JPEG $191 \mathrm{~kb}$ )

Additional file 3: Sequences used in Additional file 2. Information on sequences used in Additional file 1 is described. (XLS $25 \mathrm{~kb}$ )

Additional file 4: Novel transcripts information. Various Information on 8 novel coding transcripts are provided including average expression value, transcript length, CDS length, ORF length, transcript sequence, cds sequence, ORF sequence, primers used, and expected amplicon sizes. (XLS $90 \mathrm{~kb})$

Additional file 5: BLAST results of validated novel transcripts. The table is the BLAST output of the validated novel transcripts mapped to $P$. alecto genome. (XLS r27 kb)

\section{Competing interests}

The authors declare that they have no competing interests.

\section{Authors' contributions}

RR and GP designed the study. AKL assembled the sequence data and constructed the assembly. AKL and OE annotated the assembly. AKL and HK examined the assembled data and assessed the quality. KAK and ERN performed and interpreted the molecular studies. AKL and KAK analyzed and interpreted the expression studies. MEBJ, BRA and JST provided biological material for analysis. AKL and KAK wrote the manuscript. All authors read, edited, and approved the final manuscript.

\section{Acknowledgements}

We thank Thomas Kepler, Stephanie D'Souza, Adam Hume, Elke Muhlberger, Jenna Kelly for comments and discussion on the project. We also thank Ahhyun Kim for the illustration of a bat in Fig 1a. This work was funded by the Defense Threat Reduction Agency (DTRA) grant HDTRA1-14-1-0016 and the training program in computational biology 5T32GM082797-07. The findings and conclusions in this report are those of the authors and do not necessarily represent the views of the Centers for Disease Control and Prevention or the U.S. Army.

\section{Author details}

${ }^{1}$ Department of Biomedical Informatics, Columbia University College of Physicians and Surgeons, 1130 St. Nicholas Ave, New York, USA. ${ }^{2}$ Department of Systems Biology, Columbia University College of Physicians and Surgeons,
1130 St. Nicholas Ave, New York, USA. ${ }^{3}$ United States Army Medical Research Institute for Infectious Disease, Center for Genome Sciences, 1425 Porter St, $21702 \mathrm{Ft}$ Detrick, USA. ${ }^{4}$ Centers for Disease Control and Prevention, Viral Special Pathogens Branch, 1600 Clifton Rd. NE, 30333 Atlanta, USA. ${ }^{5}$ National Center for Biodefense and Infectious Disease, George Mason University, 20110 Manassas, USA.

Received: 6 August 2015 Accepted: 22 October 2015

Published online: 07 December 2015

\section{References}

1. Wilson DE, Reeder DM. Mammal Species of the World: a Taxonomic and Geographic Reference. Baltimore: Johns Hopkins University Press; 2005.

2. Moratelli R, Calisher $\mathrm{CH}$. Bats and zoonotic viruses: can we confidently link bats with emerging deadly viruses? Memórias do Instituto Oswaldo Cruz. 2015;110(1):1-22.

3. Teeling EC, Madsen O, Van Den Bussche RA, de Jong WW, Stanhope MJ, Springer MS. Microbat paraphyly and the convergent evolution of a key innovation in old world rhinolophoid microbats. Proc Natl Acad Sci. 2002;99(3):1431-6.

4. Calisher $\mathrm{CH}$, Childs JE, Field HE, Holmes KV, Schountz T. Bats: important reservoir hosts of emerging viruses. Clin Microbiol Rev. 2006;19(3):531-45.

5. Young PL, Halpin K, Selleck PW, Field H, Gravel JL, Kelly MA, et al. Serologic evidence for the presence in pteropus bats of a paramyxovirus related to equine morbillivirus. Emerg Infect Dis. 1996;2(3):239.

6. Smith I, Broos A, de Jong C, Zeddeman A, Smith C, Smith G, et al. Identifying hendra virus diversity in pteropid bats. PLoS One. 2011;6(9): 25275.

7. Chua K, Bellini W, Rota P, Harcourt B, Tamin A, Lam S, et al. Nipah virus: a recently emergent deadly paramyxovirus. Science. 2000;288(5470): $1432-5$.

8. Li W, Shi Z, Yu M, Ren W, Smith C, Epstein JH, et al. Bats are natural reservoirs of sars-like coronaviruses. Science. 2005;310(5748):676-9.

9. de Groot RJ, Baker SC, Baric RS, Brown CS, Drosten C, Enjuanes L, et al. Middle east respiratory syndrome coronavirus (mers-cov): announcement of the coronavirus study group. J Virol. 2013;87(14):7790-2.

10. Swanepoel R, Smit SB, Rollin PE, Formenty P, Leman PA, Kemp A, et al. Studies of reservoir hosts for marburg virus. Emerg Infect Dis. 2007;13(12): 1847

11. Towner JS, Pourrut X, Albariño CG, Nkogue CN, Bird BH, Grard G, et al. Marburg virus infection detected in a common african bat. PLoS One. 2007:2(8):764

12. Towner JS, Amman BR, Sealy TK, Carroll SAR, Comer JA, Kemp A, et al. Isolation of genetically diverse marburg viruses from egyptian fruit bats. PLoS Pathog. 2009;5(7):1000536

13. Amman BR, Jones ME, Sealy TK, Uebelhoer LS, Schuh AJ, Bird BH, et al, Oral shedding of marburg virus in experimentally infected egyptian fruit bats (rousettus aegyptiacus). J Wildl Dis. 2015;51(1):113-24.

14. Leroy $E M$, Kumulungui $B$, Pourrut $X$, Rouquet $P$, Hassanin $A$, Yaba $P$, et al. Fruit bats as reservoirs of ebola virus. Nature. 2005;438(7068):575-6.

15. Saéz AM, Weiss S, Nowak K, Lapeyre V, Zimmermann F, Düx A, et al. Investigating the zoonotic origin of the west african ebola epidemic. EMBO Mol Med. 2015;7(1):17-23.

16. Ogawa H, Miyamoto H, Nakayama E, Yoshida R, Nakamura I, Sawa H, et al. Seroepidemiological prevalence of multiple species of filoviruses in fruit bats (eidolon helvum) migrating in africa. J Infect Dis. 2015;212 Suppl 2:S101-8. doi:10.1093/infdis/jiv063 http://www.ncbi.nlm.nih.gov/ pubmed/25786916.

17. Swanepoel R, Leman PA, Burt FJ, Zachariades NA, Braack L, Ksiazek TG, et al. Experimental inoculation of plants and animals with ebola virus. Emerg Infect Dis. 1996;2(4):321.

18. Paweska JT, van Vuren PJ, Fenton KA, Graves K, Grobbelaar AA, Moolla $\mathrm{N}$, et al. Lack of marburg virus transmission from experimentally infected to susceptible in-contact egyptian fruit bats. J Infect Dis. 2015;212 Suppl 2:S109-18. doi:10.1093/infdis/jiv132 http://www.ncbi.nIm.nih.gov/ pubmed/25838270

19. Paweska JT, Van Vuren PJ, Masumu J, Leman PA, Grobbelaar AA Birkhead M, et al. Virological and serological findings in rousettus aegyptiacus experimentally inoculated with vero cells-adapted hogan strain of marburg virus. PloS one. 2012;7(9):45479. 
20. Williamson $M$, Hooper $P$, Selleck $P$, Gleeson L, Daniels $P$, Westbury $H$, et al. Transmission studies of hendra virus (equine morbilli-virus) in fruit bats, horses and cats. Aust Vet J. 1998;76(12):813-8.

21. Williamson M, Hooper P, Selleck P, Westbury H, Slocombe R. Experimental hendra virus infectionin pregnant guinea-pigs and fruit bats (pteropus poliocephalus). J Comp Pathol. 2000;122(2):201-7.

22. Middleton D, Morrissy C, Van Der Heide B, Russell G, Braun M, Westbury $\mathrm{H}$, et al. Experimental nipah virus infection in pteropid bats (pteropus poliocephalus). J Comp Pathol. 2007;136(4):266-72

23. Field H, McCall B, Barrett J. Australian bat lyssavirus infection in a captive juvenile black flying fox. Emerg Infect Dis. 1999;5(3):438.

24. Amman BR, Carroll SA, Reed ZD, Sealy TK, Balinandi S, Swanepoel R, et al. Seasonal pulses of marburg virus circulation in juvenile rousettus aegyptiacus bats coincide with periods of increased risk of human infection. PLoS Pathog. 2012;8(10):1002877.

25. Feldmann H, Geisbert TW. Ebola haemorrhagic fever. The Lancet. 2011;377(9768):849-62.

26. Pourrut X, Souris M, Towner JS, Rollin PE, Nichol ST, Gonzalez JP, et al. Large serological survey showing cocirculation of ebola and marburg viruses in gabonese bat populations, and a high seroprevalence of both viruses in rousettus aegyptiacus. BMC Infect Dis. 2009;9(1):159.

27. Olival KJ, Islam A, Yu M, Anthony SJ, Epstein JH, Khan SA, et al. Ebola virus antibodies in fruit bats, bangladesh. Emerg Infect Dis. 2013;19(2):270.

28. Jones ME, Schuh AJ, Amman BR, Sealy TK, Zaki SR, Nichol ST, et al. Experimental inoculation of egyptian rousette bats (rousettus aegyptiacus) with viruses of the ebolavirus and marburgvirus genera. Viruses. 2015;7(7):3420-42.

29. Mammalian Genome Project. https://www.broadinstitute.org/science/ projects/mammals-models/data-release-mammaliangenome-project.

30. Zhang G, Cowled C, Shi Z, Huang Z, Bishop-Lilly KA, Fang X, et al. Comparative analysis of bat genomes provides insight into the evolution of flight and immunity. Science. 2013;339(6118):456-60.

31. Seim I, Fang X, Xiong Z, Lobanov AV, Huang Z, Ma S, et al. Genome analysis reveals insights into physiology and longevity of the brandt's bat myotis brandtii. Nat Commun. 2013;4:2212. doi:10.1038/ncomms3212 http://www.pubmedcentral.nih.gov/articlerender.fcgi?artid=3753542\& tool=pmcentrez\&rendertype=abstract. Nature Publishing Group.

32. Papenfuss AT, Baker ML, Feng ZP, Tachedjian M, Crameri G, Cowled C, et al. The immune gene repertoire of an important viral reservoir, the australian black flying fox. BMC Genomics. 2012;13(1):261.

33. Shaw TI, Srivastava A, Chou WC, Liu L, Hawkinson A, Glenn TC, et al. Transcriptome sequencing and annotation for the jamaican fruit bat (artibeus jamaicensis). PloS one. 2012;7(11):48472.

34. Glennon NB, Jabado O, Lo MK, Shaw ML. Transcriptome profiling of the virus-induced innate immune response in pteropus vampyrus and its attenuation by nipah virus interferon antagonist functions. J Virol. 2015:00302. doi:10.1128/JVI.00302-15.

35. Grabherr MG, Haas BJ, Yassour M, Levin JZ, Thompson DA, Amit I, et al. Full-length transcriptome assembly from rna-seq data without a reference genome. Nat Biotechnol. 2011;29(7):644-52.

36. Lee A, Khiabanian H, Kugelman J, Elliott O, Nagle E, Yu GY, et al. Transcriptome reconstruction and annotation of cynomolgus and african green monkey. BMC Genomics. 2014;15(1):846.

37. Robertson G, Schein J, Chiu R, Corbett R, Field M, Jackman SD, et al. De novo assembly and analysis of rna-seq data. Nat Methods. 2010;7(11): 909-12.

38. Brawand D, Soumillon M, Necsulea A, Julien P, Csárdi G, Harrigan P, et al. The evolution of gene expression levels in mammalian organs. Nature. 2011:478(7369):343-8

39. Hu ZL, Bao J, Reecy JM. Categorizer: a web-based program to batch analyze gene ontology classification categories. Online Journal of Bioinformatics. 2008;9(2):108-12.

40. Omatsu T, BakEJ, Ishii Y, Kyuwa S, Tohya Y, Akashi H, et al. Induction and sequencing of rousette bat interferon $\alpha$ and $\beta$ genes. Vet Immunol Immunopathol. 2008;124(1):169-76.

41. NCBI Eukaryotic Genomes Annotations. http://www.ncbi.nlm.nih.gov/ genome/annotation_euk/all/. Accessed date March 2015.

42. FastQC. http://www.bioinformatics.babraham.ac.uk/projects/fastqc/. Accessed date March 2015

43. Martin M. Cutadapt removes adapter sequences from high-throughput sequencing reads. EMBnet journal. 2011;17(1):10.
44. Altschul SF, Madden TL, Schäffer AA, Zhang J, Zhang Z, Miller W, et al Gapped blast and psi-blast: a new generation of protein database search programs. Nucleic Acids Res. 1997;25(17):3389-402.

45. Li W, Godzik A. Cd-hit: a fast program for clustering and comparing large sets of protein or nucleotide sequences. Bioinformatics. 2006;22(13): 1658-9.

46. TransDecoder. https://transdecoder.github.io/. Accessed date March 2015.

47. Li B, Dewey CN. Rsem: accurate transcript quantification from rna-seq data with or without a reference genome. BMC Bioinf. 2011;12(1):323.

48. R Core Team. R: A Language and Environment for Statistical Computing. Vienna, Austria: R Foundation for Statistical Computing; 2015. R Foundation for Statistical Computing. http://www.R-project.org/.

49. Robinson MD, McCarthy DJ, Smyth GK. edger: a bioconductor package for differential expression analysis of digital gene expression data. Bioinformatics. 2010;26:1

50. McCarthy DJ, Chen Y, Smyth GK. Differential expression analysis of multifactor rna-seq experiments with respect to biological variation. Nucleic Acids Res. 2012:40(10):9.

51. Alexa A, Rahnenfuhrer J. topGO: topGO: Enrichment Analysis for Gene Ontology. 2010. R package version 2.18.0 https://www.bioconductor.org/ packages/devel/bioc/vignettes/topGO/inst/doc/topGO.pdf.

52. Ashburner $M$, Ball CA, Blake JA, Botstein D, Butler H, Cherry JM, et al. Gene ontology: tool for the unification of biology. Nat Genet. 2000;25(1): 25-9.

53. Quinlan AR, Hall IM. Bedtools: a flexible suite of utilities for comparing genomic features. Bioinformatics. 2010;26(6):841-2.

54. Ye J, Coulouris G, Zaretskaya I, Cutcutache I, Rozen S, Madden TL. Primer-blast: a tool to design target-specific primers for polymerase chain reaction. BMC Bioinf. 2012;13(1):134.

\section{Submit your next manuscript to BioMed Central and take full advantage of:}

- Convenient online submission

- Thorough peer review

- No space constraints or color figure charges

- Immediate publication on acceptance

- Inclusion in PubMed, CAS, Scopus and Google Scholar

- Research which is freely available for redistribution

Submit your manuscript at www.biomedcentral.com/submit 